\title{
Synaptotagmin Binding to Botulinum Neurotoxins
}

\author{
Markel Martínez-Carranza, ${ }^{\dagger}$ Pilar Blasco, ${ }^{\ddagger}, \nabla$ Robert Gustafsson, ${ }^{\dagger} @$ Min Dong, \\ Ronnie Per-Arne Berntsson, ${ }^{\|, \perp}$ Göran Widmalm, ${ }^{*},{ }^{\ddagger}$ and Pål Stenmark ${ }^{*},, \# \mathbb{C}$ \\ ${ }^{\dagger}$ Department of Biochemistry and Biophysics, Stockholm University, SE-106 91 Stockholm, Sweden \\ ${ }^{\ddagger}$ Department of Organic Chemistry, Stockholm University, SE-106 91 Stockholm, Sweden \\ ${ }^{\S}$ Department of Urology, Boston's Children Hospital, and Department of Microbiology and Immunology and Department of \\ Surgery, Harvard Medical School, Boston, Massachusetts 02115, United States \\ "Department of Medical Biochemistry and Biophysics, Umeå University, SE-90187 Umeå, Sweden \\ ${ }^{\perp}$ Wallenberg Centre for Molecular Medicine, Umeå University, SE-90187 Umeå, Sweden \\ \#Department of Experimental Medical Science, Lund University, SE-221 00 Lund, Sweden
}

\section{Supporting Information}

ABSTRACT: Botulinum neurotoxins (BoNTs) are exceptionally toxic proteins that cause paralysis but are also extensively used as treatment for various medical conditions. Most BoNTs bind two receptors on neuronal cells, namely, a ganglioside and a protein receptor. Differences in the sequence between the protein receptors from different species can impact the binding affinity and toxicity of the BoNTs. Here we have investigated how BoNT/B, /DC, and /G, all three toxins that utilize synaptotagmin I and II (Syt-I and SytII, respectively) as their protein receptors, bind to Syt-I and -II of mouse/rat, bovine, and human origin by isothermal titration calorimetry analysis. BoNT/G had the highest affinity for human Syt-I, and BoNT/DC had the highest

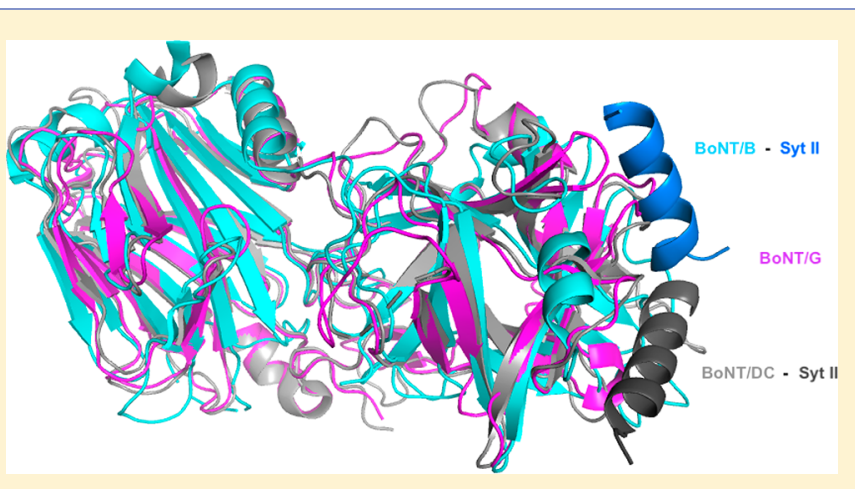
affinity for bovine Syt-II. As expected, BoNT/B, /DC, and /G showed very low levels of binding to human Syt-II. Furthermore, we carried out saturation transfer difference (STD) and STD-TOCSY NMR experiments that revealed the region of the Syt peptide in direct contact with BoNT/G, which demonstrate that BoNT/G recognizes the Syt peptide in a model similar to that in the established BoNT/B-Syt-II complex. Our analyses also revealed that regions outside the Syt peptide's toxin-binding region are important for the helicity of the peptide and, therefore, the binding affinity.

$\mathrm{W}$ ith a lethal dose $\left(\mathrm{LD}_{50}\right)$ of approximately $1 \mathrm{ng} / \mathrm{kg}$, there are few things as toxic as botulinum neurotoxins (BoNTs). ${ }^{1,2}$ As such, these toxins are classified as some of the most dangerous potential bioterrorism agents in the world. The high potency of BoNTs arises from their ability to block neurons, which leads to muscle paralysis in the host. Despite their toxicity, BoNTs are widely used to treat a number of human diseases, as well as for cosmetic purposes. 3,4

There are seven classic serotypes of BoNT, denominated BoNT/A-G. ${ }^{5-8}$ Recently, several novel toxins with unique properties have been discovered, although much remains to be studied regarding the functions and species specificity of these new toxins (known as BoNT/X, /En, and /Wo). ${ }^{8-12}$ All serotypes share the same overall structure but differ in their preferred receptors and substrates. In addition to the classic distinct serotypes, there are also a number of subtypes and mosaic serotypes, e.g., BoNT/A1-A8 and BoNT/DC. ${ }^{13-15}$ BoNTs consist of a light chain ( $\mathrm{LC}, \sim 50 \mathrm{kDa})$ that is the proteolytically active part of the toxin and a heavy chain ( $\mathrm{HC}$, $\sim 100 \mathrm{kDa}$ ), which is linked by a disulfide bridge to the LC. The $\mathrm{HC}$ can further be divided into the N-terminal membrane translocation domain $\left(\mathrm{H}_{\mathrm{N}}, \sim 50 \mathrm{kDa}\right)$ and the receptor-binding domain $\left(\mathrm{H}_{\mathrm{C}}, \sim 50 \mathrm{kDa}\right)$. The receptor-binding domain itself has two subdomains, $\mathrm{H}_{\mathrm{CC}}$ and $\mathrm{H}_{\mathrm{CN}}{ }^{16}$ The architecture of the botulinum neurotoxins is represented in Figure 1.

BoNTs bind to neuronal cells by receptors consisting of specific membrane proteins as well as gangliosides. In general, BoNTs may bind to gangliosides initially, followed by subsequent binding to a specific membrane protein in a "double-receptor" model. ${ }^{18-20}$ Notably, it has been proposed that BoNT/C does not bind to any protein receptor but instead binds to two gangliosides. ${ }^{21,22}$ Furthermore, we have proposed that BoNT/DC might have a third independent binding site, a hydrophobic loop anchoring the toxin to the lipid bilayer. ${ }^{23}$ This is also supported by recent studies of BoNT/B, /G, and/DC. ${ }^{24,25}$ Previous studies have established that BoNT/B, /DC, and /G utilize homologous synaptic

Received: June 28, 2019

Revised: December 6, 2019

Published: December 6, 2019 


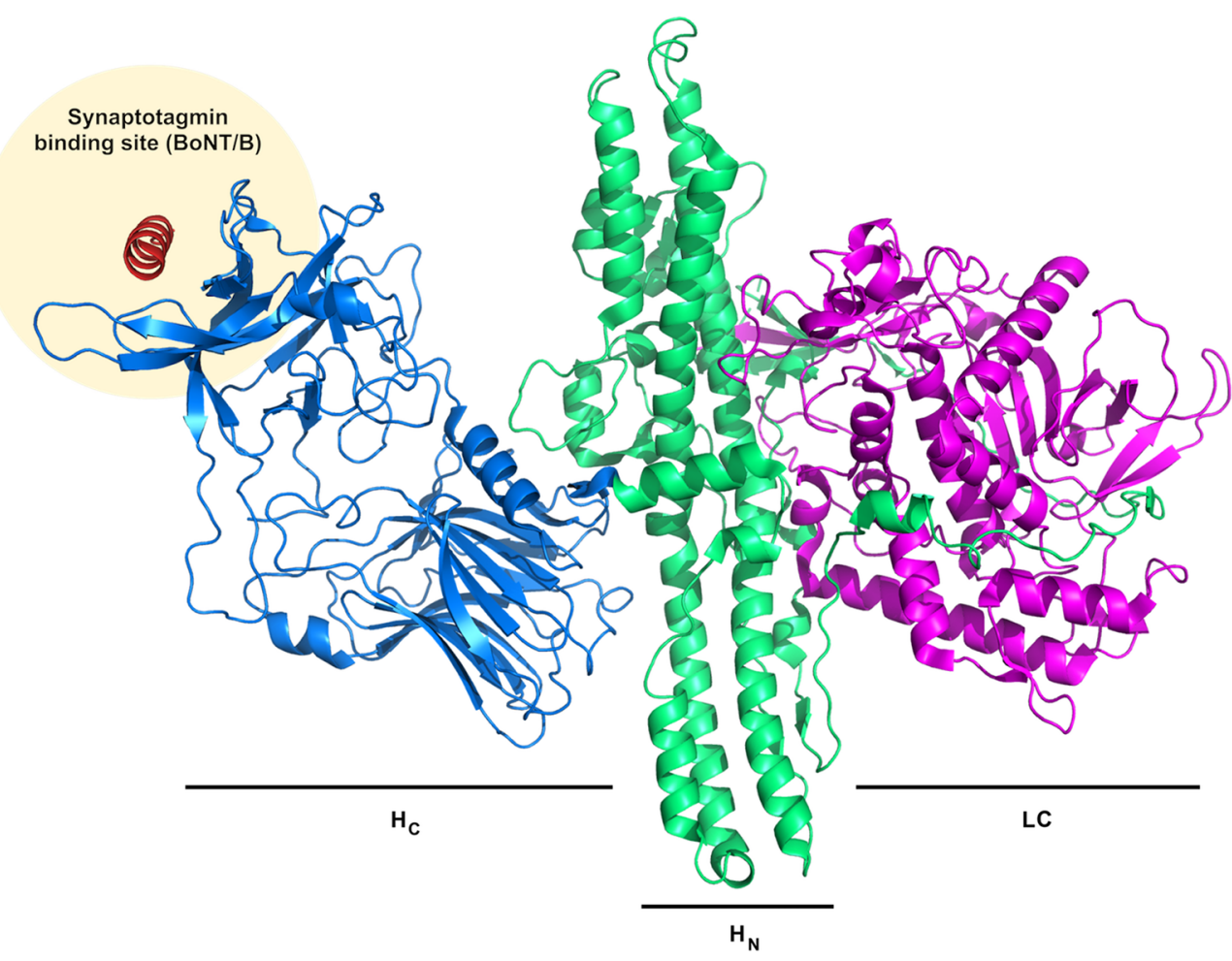

Figure 1. Crystal structure of BoNT/B together with a synaptotagmin II peptide (PDB entry $2 \mathrm{NP0}) .{ }^{17}$ The light chain (LC) is colored magenta, the translocation domain lime green $\left(\mathrm{H}_{\mathrm{N}}\right)$, the receptor-binding domain blue $\left(\mathrm{H}_{\mathrm{C}}\right)$, and the synaptotagmin peptide red.

vesicle proteins synaptotagmin I and II (Syt-I and Syt-II, respectively) as their protein receptors. ${ }^{26-31}$ BoNT/A utilizes another synaptic vesicle protein SV2 as its receptor. ${ }^{32,33}$ BoNT/D, /E, and possibly serotype /F also utilize SV2 as their receptor. ${ }^{34-38}$ As of yet, it is not known which receptors are utilized by BoNT/X, /En, or /Wo. ${ }^{8,9,39}$

BoNTs act on a number of different vertebrates. An interesting question has been the one regarding species dependence; to what extent does the sequence variation in the protein receptors in different species influence the binding by the BoNT serotypes? We and others have previously shown that there is a dramatic effect on binding of BoNT/B to Syt-II depending on whether the experiment is carried out with human or rat Syt-II. The binding affinity of BoNT/B is substantially weakened for human Syt-II compared to that of the rat and mouse counterpart. ${ }^{28,40}$ This remarkable difference is due to a single-amino acid difference, namely Phe54 in rat (and mouse), which has been substituted with a Leu in human Syt-II. It is also worth noting that BoNT/DC is a common cause of botulism in cattle, which led us to include bovine SytII in this study. ${ }^{41}$ In addition, we have recently engineered a BoNT/B variant with a high affinity for human Syt-II to improve the clinical efficacy of BoNT/B. ${ }^{42,43}$

Here we analyzed the binding of BoNT/B, /DC, and /G to human/bovine Syt-I, human Syt-II, rat/mouse Syt-II, and bovine Syt-II. In addition, in the absence of crystals for highresolution structural studies of BoNT/G together with Syt-II, we performed saturation transfer difference nuclear magnetic resonance (STD NMR) studies with BoNT/G- $\mathrm{H}_{\mathrm{C}}$ and peptides corresponding to the toxin-binding site in rat Syt-II, revealing molecular details of receptor recognition.

\section{MATERIALS AND METHODS}

Peptides and Protein Constructs. The BoNT/B- $\mathrm{H}_{\mathrm{C}}$, BoNT/DC- $\mathrm{H}_{\mathrm{C}}$, and BoNT/G- $\mathrm{H}_{\mathrm{C}}$ constructs were used as previously described. ${ }^{20,23,44}$ Peptides corresponding to the toxin-binding site in human Syt-I, rat Syt-II, human Syt-II, and bovine Syt-II were synthesized (>95\% pure) by JPT Peptide Technologies $\mathrm{GmbH}$ and GenScript, on the basis of previous studies that identified the binding region. ${ }^{17,28,45}$

Protein Expression and Purification. BoNT/B- $\mathrm{H}_{\mathrm{C}}$ BoNT/DC- $\mathrm{H}_{\mathrm{C}}$, and BoNT/G- $\mathrm{H}_{\mathrm{C}}$ were expressed and purified as previously described for BoNT/B. ${ }^{20}$ Briefly, they were expressed in the BL21 strain of Escherichia coli and grown in terrific broth at $37{ }^{\circ} \mathrm{C}$ to an $\mathrm{OD}_{600}$ of $\sim 1.5$. The culture temperature was subsequently decreased to $20{ }^{\circ} \mathrm{C}$, and expression was induced by the addition of isopropyl 1-thio$\beta$-D-galactopyranoside with a final concentration of $1 \mathrm{mM}$, and the culture allowed to grow overnight $(\sim 16 \mathrm{~h})$, before the cells were harvested and frozen at $-80{ }^{\circ} \mathrm{C}$. The proteins were purified in a two-step purification procedure, with immobilized metal affinity chromatography being the first step and sizeexclusion chromatography being the second step. The final buffer of the protein consisted of $20 \mathrm{mM} \mathrm{KP}_{\mathrm{i}}(\mathrm{pH} 7.0)$ and 150 $\mathrm{mM} \mathrm{NaCl}$.

Isothermal Titration Calorimetry. Binding of synaptotagmin-derived peptides to the binding domain of the $/ \mathrm{B}$, /DC, and /G serotypes of BoNT was assessed via isothermal titration calorimetry on an ITC200 instrument (GE Healthcare) at $25^{\circ} \mathrm{C}$ and $1000 \mathrm{rpm}$. An aliquot of $200 \mu \mathrm{L}$ of either serotype (with a typical concentration of $15-30 \mu \mathrm{M}$ ) was added to the sample cell. Binding was measured upon the addition of Syt peptides in a stepwise manner, typically 16 injections of $2.5 \mu \mathrm{L}$ each, at a concentration that was $10-15$ times the protein concentration in the sample cell. The first 


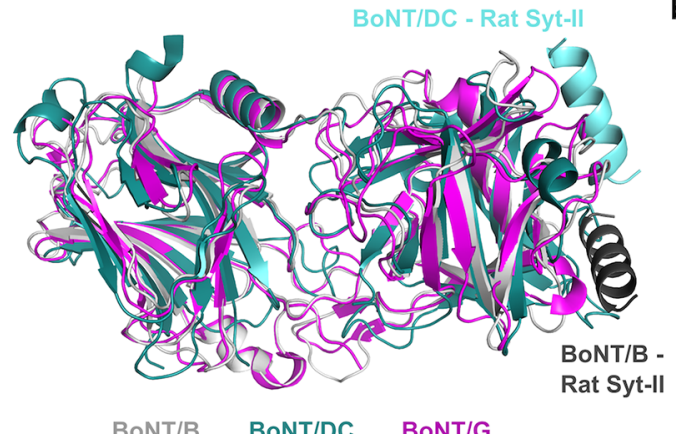

b

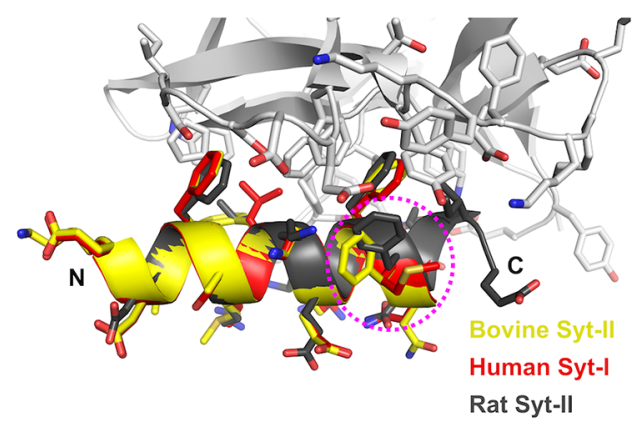

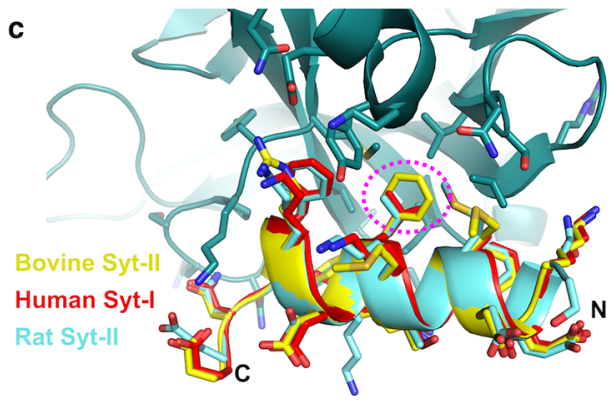

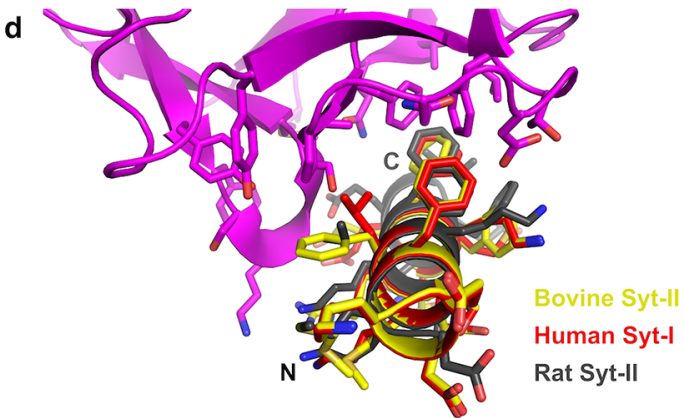

Figure 2. Structural overview of the $\mathrm{H}_{\mathrm{C}} \mathrm{s}$ of BoNT/B (gray), BoNT/DC (teal), and BoNT/G (magenta) binding to different isoforms of Syt. (a) Cartoon representation of the three BoNT serotypes with both rat Syt-II peptides (dark gray, PDB entry $2 \mathrm{NM1}{ }^{45}$ ) bound to BoNT/B and rat SytII peptide (light blue, PDB entry 4ISR ${ }^{23}$ ) bound to BoNT/DC visualized. (b-d) Structural alignment of bovine Syt-II (yellow), human Syt-I (red), and rat Syt-II (dark gray) binding to (b) BoNT/B, (c) BoNT/DC, and (d) BoNT/G (PDB entry 2VXR ${ }^{44}$ ). Variations of sequence between the synaptotagmin species and isoforms that are believed to be important for the observed differences in binding affinity are highlighted by a pink circle.

titration was set to $0.5 \mu \mathrm{L}$ and was subsequently deleted in the data analysis. A minimum of two titrations per sample were performed, and affinity and standard deviation values were calculated using Origin software.

Structural Models. The structures of the receptor-binding domains of BoNT/B complexed with rat Syt-II and BoNT/DC complexed to rat Syt-II and to human Syt-I are available in the PDB (entries 2NM1, 4ISR, and 4ISQ respectively). For BoNT/DC, the model of bovine Syt-II was made in silico using rat Syt-II from the structure of the BoNT/DC-Syt-II complex as a reference, to visualize possible reasons for the differences in binding affinity. Rotamers of the mutated residues were chosen so that they were in a conformation similar to that in the structure determined by X-ray crystallography. For BoNT/ $B$, the structures of the human Syt-I peptide (from the BoNT/ DC complex structure) and the modeled bovine Syt-II were superimposed on the rat Syt-II peptide bound to BoNT/B. Similarly, for BoNT/G, the rat Syt-II peptide structure from the BoNT/B crystal structure was used as a starting model, and the peptides were moved $\sim 3 \AA$ to take the small differences between BoNT/B and /G into account to accommodate them in the binding groove.

NMR Spectroscopy. The peptide rat Syt-II sample was prepared as a $2.5 \mathrm{mM} \mathrm{D} \mathrm{D}_{2} \mathrm{O}$ buffer solution $[20 \mathrm{mM} \mathrm{KP}$ ind $150 \mathrm{mM} \mathrm{NaCl}(\mathrm{pH} \mathrm{7})]$ and transferred to a $3 \mathrm{~mm}$ NMR tube. In the case of the truncated version of rat Syt-II (trunc rat SytII), a $4 \mathrm{mM}$ buffer solution of the peptide was used. NMR experiments were carried out at $25^{\circ} \mathrm{C}$ where the temperature had been calibrated with a sample of neat deuterated methanol. ${ }^{46}{ }^{1} \mathrm{H}$ and ${ }^{13} \mathrm{C}$ NMR chemical shifts were referenced to external 3-trimethylsilyl-(2,2,3,3- $\left.{ }^{2} \mathrm{H}_{4}\right)$-propanoate (TSP, $\delta_{\mathrm{H}}$ $0.00)$ and 1,4-dioxane in $\mathrm{D}_{2} \mathrm{O}\left(\delta_{\mathrm{C}} 67.40\right)$. For assignments of
NMR resonances, standard one-dimensional ${ }^{1} \mathrm{H}$ NMR, twodimensional ${ }^{1} \mathrm{H}-{ }^{13} \mathrm{C} \mathrm{HSQC},{ }^{1} \mathrm{H}-{ }^{1} \mathrm{H}$ TOCSY (mixing times of $30,60,90$, and $120 \mathrm{~ms}$ ), ${ }^{1} \mathrm{H}-{ }^{13} \mathrm{C} \mathrm{HMBC}$, and ${ }^{1} \mathrm{H}-{ }^{1} \mathrm{H}$ NOESY (mixing times of 120 and $300 \mathrm{~ms}$ ) experiments were carried out on a $500 \mathrm{MHz}$ Bruker AVANCE spectrometer equipped with a TCI Z-Gradient cryoprobe. STD ${ }^{47}$ and STD-TOCSY ${ }^{48}$ NMR experiments with rat Syt-II in the presence of BoNT/G$\mathrm{H}_{\mathrm{c}}$ were performed with a 1:100 protein:peptide ratio $(18 \mu \mathrm{M}$ protein and $2 \mathrm{mM}$ peptide). On- and off-resonance frequencies were set at -0.5 and $60 \mathrm{ppm}$, respectively, employing a $2 \mathrm{~s}$ irradiation time. STD NMR experiments were performed with 7200 scans and $16 \mathrm{~K}$ data points; STD-TOCSY experiments employed 48 scans and $2048 \times 512$ data points. For analysis of the NMR spectra, CARA software was used. ${ }^{49}$ The chemical shift index $(\mathrm{CSI})^{50}$ was used for analysis of the secondary structure of the free peptides in solution.

\section{RESULTS}

Binding of BoNT/B, /DC, and /G to Syt-I and Syt-II. BoNT/DC and BoNT/B bind to Syt with structurally distinct binding sites (Figure 2a). ${ }^{23}$ In both cases, human Syt-II binds with a much lower affinity than rat/mouse Syt-II. ${ }^{28}$

To further investigate the species dependence of Syt binding to $\mathrm{BoNT} / \mathrm{B}, / \mathrm{DC}$, and $/ \mathrm{G}$, we performed isothermal titration calorimetry (ITC) experiments. The $\mathrm{H}_{\mathrm{C}}$ domain of the three serotypes was titrated with peptides of the toxin-binding domains of human/bovine Syt-I, human Syt-II, rat Syt-II, and bovine Syt-II (see Figure 3 for their respective sequences). The binding sites of human and bovine Syt-I are identical, and they differ from those of rat and mouse Syt-I only at position 52 (E52 and Q52, respectively). Rat Syt-II and mouse Syt-II differ from each other similarly (D52 and E52, respectively). At the 


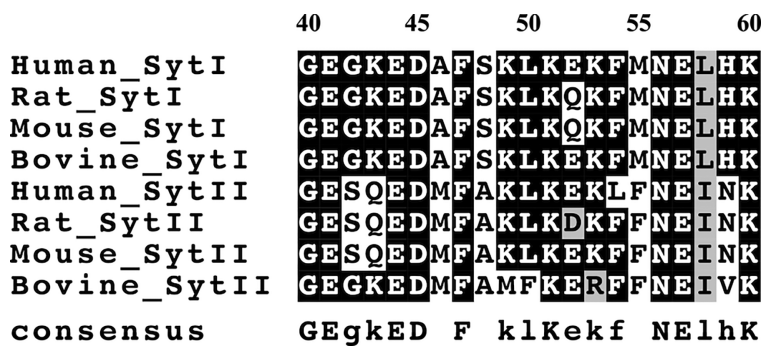

Figure 3. Multiple-sequence alignment of extracellular regions from human, rat, mouse, and bovine Syt-I and Syt-II, numbered according to the rat Syt-II frame. Black shades indicate fully conserved residues with respect to the consensus sequence, while gray shades indicate conservation between amino acids of similar chemical properties. Uppercase letters in the consensus sequence indicate a fully conserved residue across all eight sequences.

binding interface, the side chain of the residue in this position is pointing outward and is not involved in the binding to BoNT, as has been demonstrated for BoNT/DC, ${ }^{20,28}$ and on the basis of the structures of the BoNT/B-Syt-II complex, it is highly probable that this is the case for BoNT/B and BoNT/G, as well. ${ }^{17,20,45}$ Therefore, rat Syt-II binding resembles that of mouse Syt-II. All serotypes bound human Syt-I, with affinities ranging from 0.3 to $6.7 \mu \mathrm{M}$ (Table 1 and Figure S1). Only

Table 1. Values of $K_{\mathrm{D}}, \Delta H, T \Delta S$, and $\Delta G$ Determined via ITC for BoNT/B, BoNT/DC, and BoNT/G $\mathrm{H}_{\mathrm{C}}$ domains and Syt-I and Syt-II ${ }^{a}$

$\begin{array}{ccccc} & K_{\mathrm{D}}(\mu \mathrm{M}) & \begin{array}{c}\Delta H \\ (\mathrm{kcal} / \mathrm{mol})\end{array} & \begin{array}{c}\mathrm{T} \Delta S \\ (\mathrm{kcal} / \mathrm{mol})\end{array} & \begin{array}{c}\Delta G \\ (\mathrm{kcal} / \mathrm{mol})\end{array} \\ \text { Syt-I (human) } & & & & \\ \text { BoNT/B } & 0.9 \pm 0.1 & -4.4 \pm 0.5 & 3.8 \pm 0.4 & -8.2 \\ \text { BoNT/DC } & 6.7 \pm 0.2 & -6.7 \pm 3.1 & 0.3 \pm 3.2 & -7.1 \\ \text { BoNT/G } & 0.3 \pm 0.1 & -17.6 \pm 0.9 & -7.7 \pm 0.1 & -9.1 \\ \text { Syt-II (human) } & & & & \\ \text { BoNT/B } & 20 \pm 6 & -3.9 \pm 1.1 & 2.5 \pm 1.3 & -6.4 \\ \text { BoNT/DC } & \mathrm{ND} & & & \\ \text { BoNT/G } & \mathrm{ND} & & & \\ \text { Syt-II (rat) } & & & & \\ \text { BoNT/B } & 0.1 \pm 0.05 & -7.5 \pm 0.1 & 1.9 \pm 0.2 & -9.4 \\ \text { BoNT/DC } & 3.0 \pm 0.3 & -14.5 \pm 0.4 & -6.9 \pm 0.5 & -7.6 \\ \text { BoNT/G } & 0.6 \pm 0.2 & -10.3 \pm 0.4 & -1.7 \pm 0.1 & -8.6 \\ \text { Syt-II (bovine) } & & & & \\ \text { BoNT/B } & 1.0 \pm 0.4 & -6.7 \pm 0.5 & 1.5 \pm 0.7 & -8.2 \\ \text { BoNT/DC } & 0.8 \pm 0.3 & -17.3 \pm 5.0 & -8.9 \pm 5.1 & -8.4 \\ \text { BoNT/G } & 1.9 \pm 0.1 & -5.8 \pm 0.3 & 2.0 \pm 0.3 & -7.8\end{array}$

${ }^{a}$ The errors reported are the standard deviations of at least two independent measurements.

BoNT/B bound to human Syt-II ( $K_{\mathrm{D}}$ of $\left.20 \pm 6 \mu \mathrm{M}\right)$, whereas the binding to BoNT/DC and BoNT/G was too weak to be directly measured by ITC. Rat/mouse Syt-II had affinities of $0.1-3.0 \mu \mathrm{M}$, and bovine Syt-II had $K_{\mathrm{D}}$ values in the range of $0.8-1.9 \mu \mathrm{M}$.

With regard to enthalpic and entropic contributions, all binding events were enthalpy-driven (Table 1). However, it should be noted that the enthalpic and entropic values reported here come from measurements at only one temperature. Some binding events, e.g., all of the Syt binding to BoNT/B, had positive entropy contributions. For BoNT/DC, we lack stable plateau levels in the titrations, which makes the $\Delta H$ values, and especially the derived $\Delta S$ values, uncertain.

To validate our conclusions from the ITC interaction studies and the structural analysis, we designed a mutant bovine Syt-II F50L peptide and determined its binding constants with BoNT/B-H $\mathrm{C}_{\mathrm{C}}$ and BoNT/DC- $\mathrm{H}_{\mathrm{C}}$ as described previously. The results are shown in Figure 4. The binding affinities for BoNT/



Figure 4. $K_{\mathrm{D}}$ values of wild-type bovine Syt-II (colored gray, data taken from Table 1) and mutant peptide bovine Syt-II F50L (colored white) measured by ITC with BoNT/B- $\mathrm{H}_{\mathrm{C}}$ and BoNT/DC- $\mathrm{H}_{\mathrm{C}}$.

B- $\mathrm{H}_{\mathrm{C}}$ and BoNT/DC- $\mathrm{H}_{\mathrm{C}}$ were measured to be $180 \mathrm{nM}$ and $2.21 \mu \mathrm{M}$, respectively. Enthalpic and entropic contributions are shown in Table S2 together with representative ITC curves in Figure S2.

Peptide Recognition Using STD NMR Spectroscopy. The complexes of Syt binding to BoNT/B and BoNT/DC have been previously determined. ${ }^{17,20,23,28,45}$ However, our ample attempts to determine the crystal structure of BoNT/G$\mathrm{H}_{\mathrm{C}}$ in complex with a Syt receptor have been unsuccessful. Facing the lack of success in crystallization of the peptide/ protein complex of BoNT/G, we used NMR spectroscopy to gain insights into the molecular recognition process. The rat Syt-II peptide was characterized in the free state, and ${ }^{1} \mathrm{H} \alpha$ and ${ }^{13} \mathrm{C} \alpha$ NMR chemical shift assignments at $25{ }^{\circ} \mathrm{C}$ were carried out (Table S1). Subsequently, $\mathrm{STD}^{47}$ and STD-TOCSY ${ }^{48}$ experiments were conducted to provide information about the epitope of the peptide in direct contact with BoNT/G-H $\mathrm{H}_{\mathrm{C}}$. The STD NMR spectrum of rat Syt-II clearly showed binding to the protein (Figure 5), where STD signals could be identified as originating from the 21-amino acid peptide (Figure 5a). Strong STD effects ( $>80 \%$ of the relative intensity) were observed for the amino acids corresponding to protein residues L50, K53, and I58, medium contribution (80-50\% relative intensity) for residues A48 and $\mathrm{K51}$, and weak effects (<50\%) for residues E44, D52, and E57 (Figure 5b). An STD-TOCSY experiment verified some of the mentioned amino acids and also identified spin systems from F47, F54, F55, and N56 residues receiving magnetization, transferred from the protein (Figure 5c). Having identified the binding site of rat Syt-II, analysis was carried out for a truncated version, i.e., truncated 


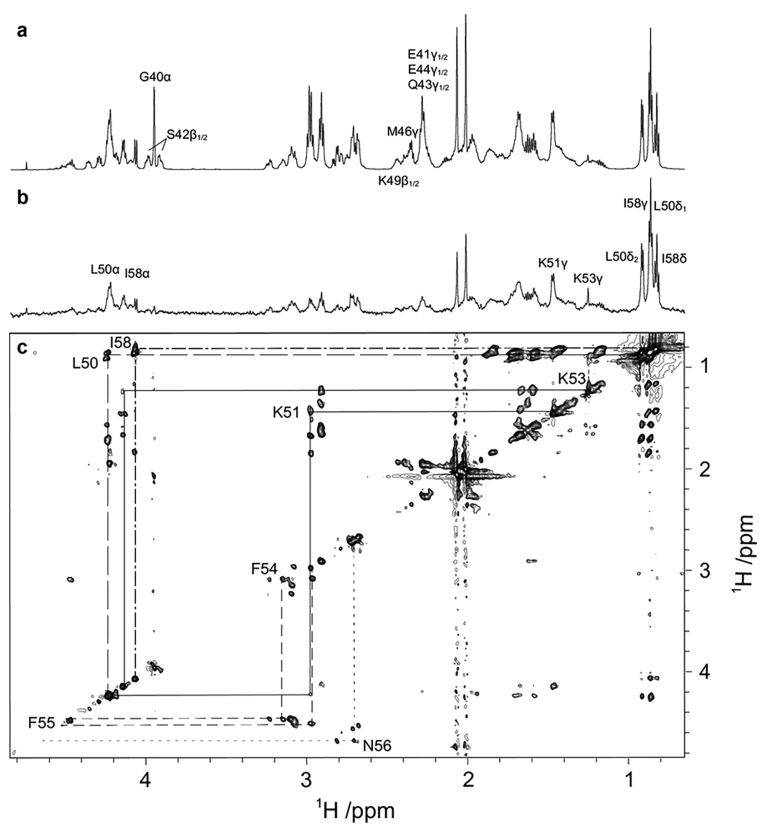

Figure 5. (a) ${ }^{1} \mathrm{H}$ NMR spectrum of the rat Syt-II peptide and (b) STD NMR and (c) STD-TOCSY NMR spectra in the presence of BoNT/G-H $\mathrm{H}_{\mathrm{C}}$. Pertinent amino acid signals are annotated.

rat Syt-II, spanning K49-N59 in Syt-II of the full-length peptide. However, STD effects were not detected upon addition of the protein, showing the absence of marked binding (data not shown).
To acquire information about the secondary structure of the rat Syt-II peptide (G40-K60), the ${ }^{1} \mathrm{H}$ NMR chemical shifts of the $\alpha$-protons were compared to reference chemical shifts reported by Wishart and co-workers where deviations of $>0.1$ ppm are compiled as index values of -1 if the chemical shift is less than the reference value, +1 if it is larger, and 0 if it is less than $\pm 0.1 \mathrm{ppm}$, corresponding to $\alpha$-helix, $\beta$-sheet, and random coil, respectively. The methodology is termed the CSI. ${ }^{50-52}$ For the rat Syt-II peptide, it is evident that to a large extent the peptide is an $\alpha$-helix (Figure $6 \mathrm{c}$ and Table S1) and that STD effects occur as a function of the secondary structure of the peptide, i.e., with 3.6 amino acids per turn of the helix, at F47, L50, K51, and K53-E57, whereas this is not pronounced for the truncated peptide (Figure $6 \mathrm{~d}$ ). These results agree with the point mutant studies performed by Willjes et al. ${ }^{53}$ For both peptides, an "end effect" is observed for I59 where the CSI is opposite in magnitude to those of the $\alpha$-helical structure (Figures 5d and 6c). ${ }^{50}$ With the gained information, the contact region for the $\alpha$-helical structure of the rat Syt-II peptide was subsequently modeled on the basis of that of the BoNT/B-H ${ }_{\mathrm{C}}$ complex (Figure 6e). ${ }^{45}$

\section{DISCUSSION}

Our interest in Syt binding to BoNT was heightened by our previous findings that human Syt-II could not effectively be utilized by BoNT/B, /DC, or/G for entry into neuronal cells, ${ }^{28}$ and F54L has previously been described to be the main cause for this. ${ }^{28,40}$ This finding raised further questions regarding the species dependence of BoNTs. Are there further sequence discrepancies in synaptotagmin between species that are

a Amino acid GLY GLU SER GLN GLU ASP MET PHE ALA LYS LEU LYS ASP LYS PHE PHE ASN GLU ILE ASN LYS

b Rat Syt-II

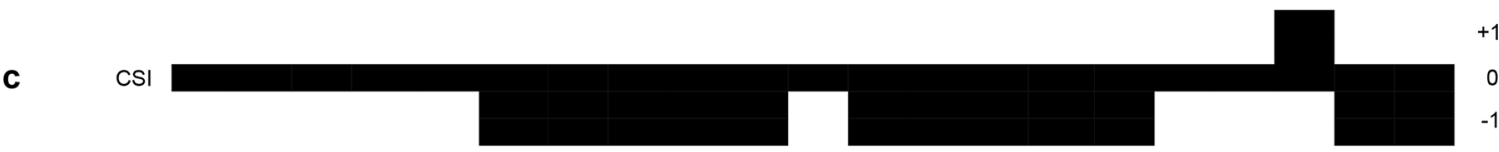

trunc Rat Syt-II

d $\quad \operatorname{csl}$

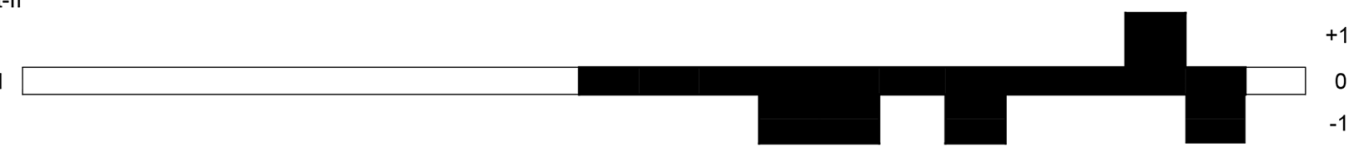

e

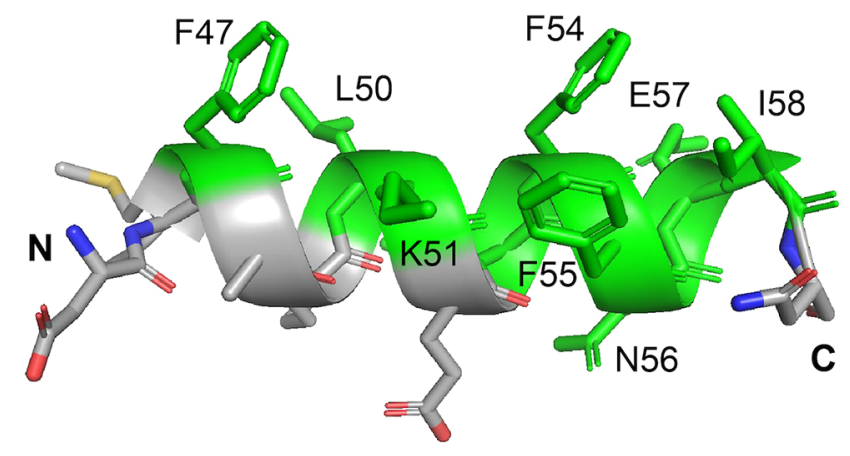

Figure 6. Rat Syt-II peptide: (a) sequence and amino acid numbering, (b) residues showing the strong STD effect (>80\%) and the STD-TOCSY contribution (green), (c) chemical shift index (CSI) for identification of the rat Syt-II peptide secondary structure, (d) CSI analysis of the truncated rat Syt-II peptide, and (e) visualization of the contact region (green) between the rat Syt-II peptide and BoNT/G-H $\mathrm{H}_{\mathrm{C}}$ protein based on STD NMR experiments using the corresponding $\alpha$-helical portion of the crystal structure of BoNT/B. 
detrimental to BoNT binding such as the F54L mutation between rat and human Syt-II?

BoNT/B binds the strongest $\left(K_{\mathrm{D}}=0.1 \mu \mathrm{M}\right)$ to rat $/$ mouse Syt-II. This affinity is slightly weaker than the previously reported dissociation constant of $38 \mathrm{nM}$ for this interaction. ${ }^{45}$ The difference in the reported affinities is likely due to different experimental conditions; e.g., the previous study used a GSTtagged Syt-II peptide, whereas we used a nontagged variant of the rat Syt-II peptide. The affinity of BoNT/B for human Syt-I and bovine Syt-II is almost 1 order of magnitude lower than that for rat Syt-II. There are only a few differences in the sequence of the residues that interact with BoNT/B (Figure 3). A possible explanation for the lower affinity for human Syt-I could be the F55M mutation (colored pink in Figure 2b), which likely decreases the level of hydrophobic packing. For bovine Syt-II, we hypothesized that its lower affinity might be caused by the L50F mutation (colored pink in Figure 2b). The fit of the phenylalanine side chain seems not to be optimal in this hydrophobic pocket. To investigate this further, we designed a bovine Syt-II F50L peptide and measured its binding affinity for $\mathrm{BoNT} / \mathrm{B}-\mathrm{H}_{\mathrm{C}}$ and $\mathrm{BoNT} / \mathrm{DC}-\mathrm{H}_{\mathrm{C}}$ via ITC. The affinity for BoNT/B was enhanced 5-fold compared to that of wild-type bovine Syt-II, which confirmed our hypothesis.

In the case of BoNT/DC, bovine Syt-II is the strongest binder $\left(K_{\mathrm{D}}=0.8 \mu \mathrm{M}\right)$, but the differences in affinity for the other two peptides that bind are within the same order of magnitude (Table 1). It is likely that bovine Syt-II binds the strongest due to the F50 residue mentioned above, where both human Syt-I and rat Syt-II have a leucine (highlighted in Figure 2c). It is surmised that the presence of a phenylalanine residue at this position leads to better hydrophobic packing and, therefore, to higher affinity. Our ITC experiments with BoNT/DC- $\mathrm{H}_{\mathrm{C}}$ and bovine Syt-II F50L are coherent with this conclusion, because the binding affinity was decreased by a factor of 3 compared to wild-type bovine Syt-II. M49 might also contribute to stabilizing this hydrophobic core.

The structure of BoNT/G together with its bound protein has not yet been determined. However, the overall structure of $\mathrm{BoNT} / \mathrm{G}-\mathrm{H}_{\mathrm{C}}$ is similar to that of $\mathrm{BoNT} / \mathrm{B}-\mathrm{H}_{\mathrm{C}}$, with a highly similar binding groove for Syt, indicating that BoNT/G binds Syt in the same manner as BoNT/B (Figure 2a). ${ }^{44,53}$ To visualize the binding, a model of how BoNT/G binds rat SytII, human Syt-I, and bovine Syt-II is shown in Figure 2d, on the basis of the interface between BoNT/B-H $\mathrm{H}_{\mathrm{C}}$ and the rat SytII peptide from their crystal structure. The most conspicuous candidate for the lower affinity of bovine Syt-II is the same as that for BoNT/B, namely the L50F mutation.

Previous mutational studies support the conclusions presented above. In an assay by Jin et al., the F55A mutation in Syt-II abolished binding to BoNT/B-H $\mathrm{C}_{\mathrm{C}}$. L50A Syt-II and L50I Syt-I decreased the binding affinities for BoNT/B- $\mathrm{H}_{\mathrm{C}}$ to approximately $20 \%$ of the wild-type synaptotagmins. ${ }^{45}$ Additional mutational studies were performed by Willjes et al. ${ }^{53}$ They report complete abolishment of binding of F55A to both BoNT/B-H $\mathrm{H}_{\mathrm{C}}$ and BoNT/G-H $\mathrm{H}_{\mathrm{C}}$. Furthermore, the L50A point mutant of rat Syt-II decreased the level of binding of BoNT/B$\mathrm{H}_{\mathrm{C}}$ to $40 \%$ and the binding of BoNT/G- $\mathrm{H}_{\mathrm{C}}$ to $20 \%$.

This is in agreement with our results and our analyses of synaptotagmin binding to these toxins.

Similar interaction areas were derived from X-ray crystallography structures for rat Syt-II in the presence of BoNT/DC and $\mathrm{BoNT} / \mathrm{B}-\mathrm{H}_{\mathrm{C}}$; the second half of the peptide (C-terminal) showed spatial proximity and hydrogen bonding with residues of the protein-binding site. Thus, this peptide moiety is vital for making important contacts with the protein. It was shown by STD NMR that it is the C-terminal part of the peptide that binds to the protein, but the absence of any significant binding and interaction between the truncated rat Syt-II peptide is presumably due to lack of inherent helicity in the short peptide. ${ }^{54} \mathrm{CSI}^{50}$ analysis showed that the $\alpha$-helix propensity in the decamer was significantly reduced (Figure 6 and Table S1). These results indicate that although the first half of the peptide is not binding strongly to the protein surface it is essential for inducing a larger degree of helix formation in the peptide and consequently the ability of it to appropriately make contact and fit in the binding site.

Among the Syt species we tested, only human Syt-II showed a dramatic decrease in binding affinity for BoNT/B, /DC, and /G. Although we do not have exact values of the affinities of BoNT/DC and /G for human Syt-II (due to their low affinity, which made direct ITC measurements difficult), estimated values based on competition experiments with BoNT/DC indicate a $>100$-fold decrease in affinity when compared to rat Syt-II, corresponding well to previously reported values in the literature. ${ }^{28}$ Furthermore, even though none of the other species of Syt tested here had such a detrimental effect on the binding, it is clear that the BoNT serotypes bind with significantly different affinities for different Syt variants. This likely translates into differences in the efficiency of neuronal cell entry between species. In addition, we conclude from our NMR studies that the regions proximal to the binding site in Syt receptors are relevant for the binding.

\section{ASSOCIATED CONTENT}

\section{S Supporting Information}

The Supporting Information is available free of charge at https://pubs.acs.org/doi/10.1021/acs.biochem.9b00554.

Rat Syt-II and trunc rat Syt-II ${ }^{1} \mathrm{H} \alpha$ and ${ }^{13} \mathrm{C} \alpha$ NMR chemical shifts (Table $\mathrm{S} 1$ ), synaptotagmin binding to $\mathrm{BoNT} / \mathrm{B}-\mathrm{H}_{\mathrm{C}}, / \mathrm{DC}-\mathrm{H}_{\mathrm{C}}$, and $/ \mathrm{G}-\mathrm{H}_{\mathrm{C}}$ with representative ITC traces of the three different BoNT serotypes binding to human synaptotagmin I, rat synaptotagmin II, and bovine synaptotagmin II (Figure $S 1$ ), $K_{\mathrm{D}}, \Delta H, T \Delta S$, and $\Delta G$ values determined via ITC for $\mathrm{BoNT} / \mathrm{B}$ and $\mathrm{BoNT} / \mathrm{DC} \mathrm{H}_{\mathrm{C}}$ domains and bovine wild-type and F50L mutant Syt-II (Table S2), and bovine Syt-II F50L binding to $\mathrm{BoNT} / \mathrm{B}-\mathrm{H}_{\mathrm{C}}$ and $/ \mathrm{DC}-\mathrm{H}_{\mathrm{C}}$ with representative ITC traces (Figure S2) (PDF)

\section{Accession Codes}

BoNT/A, UniProtKB P0DPI1; BoNT/B, UniProtKB B1INP5; BoNT/C, UniProtKB P18640; BoNT/D, UniProtKB Q9QTG7; BoNT/DC, UniProtKB Q9LBR1; BoNT/E, UniProtKB A8Y875; BoNT/F, UniProtKB P30996; BoNT/ G, UniProtKB A0A0C1TVZ1; BoNT/X, UniProtKB P0DPK1; BoNT/Wo, UniProtKB A0A069CUU9; BoNT/En, UniProtKB A0A242DI27; human synaptotagmin I, UniProtKB P21579; rat synaptotagmin I, UniProtKB P21707; mouse synaptotagmin I, UniProtKB P46096; bovine synaptotagmin I, UniProtKB P48018; human synaptotagmin II, UniProtKB Q8N9I0; rat synaptotagmin II, UniProtKB P29101; mouse synaptotagmin II, UniProtKB P46097; bovine synaptotagmin II, NCBI RefSeq XP_002694302.3. 


\section{AUTHOR INFORMATION}

\section{Corresponding Authors}

*E-mail: stenmark@dbb.su.se. Telephone: +46 8-16 3729.

*E-mail: goran.widmalm@su.se. Telephone: +46 8-16 3742.

ORCID $\odot$

Göran Widmalm: 0000-0001-8303-4481

Pål Stenmark: 0000-0003-4777-3417

\section{Present Addresses}

${ }^{\circledR}$ R.G.: Department of Cell and Molecular Biology, Uppsala University, Husargatan 3, SE-75237 Uppsala, Sweden.

${ }^{\nabla}$ P.B.: Department of Chemistry, State Key Lab of Synthetic Chemistry, The University of Hong Kong, Pokfulam Road, Hong Kong.

\section{Author Contributions}

M.M.-C. and P.B. contributed equally to this work. P.S. and G.W. conceived the project and supervised the study. M.M.-C., P.B., R.G., and R.P.-A.B. performed experiments and analyzed data. M.D. provided the BoNT/DC-Hc protein.

\section{Funding}

This work was supported by grants from the Swedish Research Council (2018-03406) and the Swedish Cancer Society to P.S., by an EMBO Long-term Fellowship and Marie Curie Actions (EMBOCOFUND2010, GA-2010-267146) and the Knut and Alice Wallenberg Foundation to R.P.-A.B., by National Institutes of Health Grants 8P51OD011103-51 (to the New England Primate Research Center), 1R56AI097834-01, and R01NS080833 to M.D., and by the Swedish Research Council (2013-4859 and 2017-03703) and The Knut and Alice Wallenberg Foundation to G.W.

\section{Notes}

The authors declare no competing financial interest.

\section{ACKNOWLEDGMENTS}

The authors thank the Biomolecule Production Core at the NERCE (New England Regional Center for Excellence, NIAID U54 AI057159) for their assistance. The authors acknowledge GE Healthcare for the use of the ITC200 machine in their demo lab.

\section{ABBREVIATIONS}

BoNT, botulinum neurotoxin; LC, light chain; HC, heavy chain; Syt-I, synaptotagmin I; Syt-II, synaptotagmin-II; ITC, isothermal titration calorimetry; NMR, nuclear magnetic resonance; HSQC, heteronuclear single-quantum coherence; NOESY, nuclear Overhauser spectroscopy; STD, saturation transfer difference; TOCSY, total correlation spectroscopy; PDB, Protein Data Bank.

\section{REFERENCES}

(1) Gill, D. M. (1982) Bacterial toxins: A table of lethal amounts. Microbiol. Rev. 46, 86-94.

(2) Arnon, S. S., Schechter, R., Inglesby, T. V., Henderson, D. A., Bartlett, J. G., Ascher, M. S., Eitzen, E., Fine, A. D., Hauer, J., Layton, M., Lillibridge, S., Osterholm, M. T., O’Toole, T., Parker, G., Perl, T. M., Russell, P. K., Swerdlow, D. L., Tonat, K., and for the Working Group on Civilian Biodefense (2001) Botulinum toxin as a biological weapon: Medical and public health management. JAMA, J. Am. Med. Assoc. 285, 1059-1070.

(3) Dolly, J. O., Lawrence, G. W., Meng, J., Wang, J., and Ovsepian, S. V. (2009) Neuro-exocytosis: botulinum toxins as inhibitory probes and versatile therapeutics. Curr. Opin. Pharmacol. 9, 326-335.
(4) Johnson, E. A. (1999) Clostridial Toxins as Therapeutic Agents: Benefits of Nature's Most Toxic Proteins. Annu. Rev. Microbiol. 53, $551-575$.

(5) Barash, J. R., and Arnon, S. S. (2014) A novel strain of clostridium botulinum that produces type $\mathrm{B}$ and type $\mathrm{H}$ botulinum toxins. J. Infect. Dis. 209, 183-191.

(6) Montal, M. (2010) Botulinum Neurotoxin: A Marvel of Protein Design. Annu. Rev. Biochem. 79, 591-617.

(7) Schiavo, G., Matteoli, M., and Montecucco, C. (2000) Neurotoxins affecting neuroexocytosis. Physiol. Rev. 80, 717-766.

(8) Zhang, S., Lebreton, F., Mansfield, M. J., Miyashita, S. I., Zhang, J., Schwartzman, J. A., Tao, L., Masuyer, G., Martínez-Carranza, M., Stenmark, P., Gilmore, M. S., Doxey, A. C., and Dong, M. (2018) Identification of a Botulinum Neurotoxin-like Toxin in a Commensal Strain of Enterococcus faecium. Cell Host Microbe 23, 169-176.

(9) Zhang, S., Masuyer, G., Zhang, J., Shen, Y., Lundin, D., Henriksson, L., Miyashita, S. I., Martínez-Carranza, M., Dong, M., and Stenmark, P. (2017) Identification and characterization of a novel botulinum neurotoxin. Nat. Commun. 8, 14130.

(10) Zornetta, I., Azarnia Tehran, D., Arrigoni, G., Anniballi, F., Bano, L., Leka, O., Zanotti, G., Binz, T., and Montecucco, C. (2016) The first non Clostridial botulinum-like toxin cleaves VAMP within the juxtamembrane domain. Sci. Rep. 6, 30257.

(11) Mansfield, M. J., Adams, J. B., and Doxey, A. C. (2015) Botulinum neurotoxin homologs in non-Clostridium species. FEBS Lett. 589, 342-348.

(12) Masuyer, G., Zhang, S., Barkho, S., Shen, Y., Henriksson, L., Košenina, S., Dong, M., and Stenmark, P. (2018) Structural characterisation of the catalytic domain of botulinum neurotoxin $\mathrm{X}$ - High activity and unique substrate specificity. Sci. Rep. 8, 4518.

(13) Carter, A. T., Paul, C. J., Mason, D. R., Twine, S. M., Alston, M. J., Logan, S. M., Austin, J. W., and Peck, M. W. (2009) Independent evolution of neurotoxin and flagellar genetic loci in proteolytic Clostridium botulinum. BMC Genomics 10, 115.

(14) Hill, K. K., Xie, G., Foley, B. T., and Smith, T. J. (2015) Genetic diversity within the botulinum neurotoxin-producing bacteria and their neurotoxins. Toxicon 107, 2-8.

(15) Peck, M. W., Smith, T. J., Anniballi, F., Austin, J. W., Bano, L., Bradshaw, M., Cuervo, P., Cheng, L. W., Derman, Y., Dorner, B. G., Fisher, A., Hill, K. K., Kalb, S. R., Korkeala, H., Lindström, M., Lista, F., Lúquez, C., Mazuet, C., Pirazzini, M., Popoff, M. R., Rossetto, O., Rummel, A., Sesardic, D., Singh, B. R., and Stringer, S. C. (2017) Historical perspectives and guidelines for botulinum neurotoxin subtype nomenclature. Toxins 9, 38.

(16) Dong, M., Masuyer, G., and Stenmark, P. (2019) Botulinum and Tetanus Neurotoxins. Annu. Rev. Biochem. 88, 811-837.

(17) Chai, Q., Arndt, J. W., Dong, M., Tepp, W. H., Johnson, E. A., Chapman, E. R., and Stevens, R. C. (2006) Structural basis of cell surface receptor recognition by botulinum neurotoxin B. Nature 444, 1096-1100.

(18) Montecucco, C. (1986) How do tetanus and botulinum toxins bind to neuronal membranes? Trends Biochem. Sci. 11, 314-317.

(19) Hamark, C., Berntsson, R. P. A., Masuyer, G., Henriksson, L. M., Gustafsson, R., Stenmark, P., and Widmalm, G. (2017) Glycans confer specificity to the recognition of ganglioside receptors by botulinum neurotoxin A. J. Am. Chem. Soc. 139, 218-230.

(20) Berntsson, R. P. A., Peng, L., Dong, M., and Stenmark, P. (2013) Structure of dual receptor binding to botulinum neurotoxin B. Nat. Commun. 4, 2058.

(21) Strotmeier, J., Gu, S., Jutzi, S., Mahrhold, S., Zhou, J., Pich, A., Eichner, T., Bigalke, H., Rummel, A., Jin, R., and Binz, T. (2011) The biological activity of botulinum neurotoxin type $\mathrm{C}$ is dependent upon novel types of ganglioside binding sites. Mol. Microbiol. 81, 143-156.

(22) Tsukamoto, K., Kohda, T., Mukamoto, M., Takeuchi, K., Ihara, H., Saito, M., and Kozaki, S. (2005) Binding of Clostridium botulinum type $\mathrm{C}$ and $\mathrm{D}$ neurotoxins to ganglioside and phospholipid: Novel insights into the receptor for clostridial neurotoxins. J. Biol. Chem. 280, 35164-35171. 
(23) Berntsson, R. P. A., Peng, L., Svensson, L. M., Dong, M., and Stenmark, P. (2013) Crystal structures of botulinum neurotoxin dc in complex with its protein receptors synaptotagmin i and II. Structure $21,1602-1611$

(24) Zhang, S., Berntsson, R. P. A., Tepp, W. H., Tao, L., Johnson, E. A., Stenmark, P., and Dong, M. (2017) Structural basis for the unique ganglioside and cell membrane recognition mechanism of botulinum neurotoxin DC. Nat. Commun. 8, 1637.

(25) Stern, D., Weisemann, J., Le Blanc, A., von Berg, L., Mahrhold, S., Piesker, J., Laue, M., Luppa, P. B., Dorner, M. B., Dorner, B. G., and Rummel, A. (2018) A lipid-binding loop of botulinum neurotoxin serotypes $\mathrm{B}, \mathrm{DC}$ and $\mathrm{G}$ is an essential feature to confer their exquisite potency. PLoS Pathog. 14, No. e1007048.

(26) Dong, M., Richards, D. A., Goodnough, M. C., Tepp, W. H., Johnson, E. A., and Chapman, E. R. (2003) Synaptotagmins I and II mediate entry of botulinum neurotoxin B into cells. J. Cell Biol. 162, $1293-1303$.

(27) Rummel, A., Karnath, T., Henke, T., Bigalke, H., and Binz, T. (2004) Synaptotagmins I and II act as nerve cell receptors for botulinum neurotoxin G. J. Biol. Chem. 279, 30865-30870.

(28) Peng, L., Berntsson, R. P. A., Tepp, W. H., Pitkin, R. M., Johnson, E. A., Stenmark, P., and Dong, M. (2012) Botulinum neurotoxin D-C uses synaptotagmin I and II as receptors, and human synaptotagmin II is not an effective receptor for type B, D-C and G toxins. J. Cell Sci. 125, 3233-3242.

(29) Nishiki, T., Kamata, Y., Nemoto, Y., Omori, A., Ito, T., Takahashi, M., and Kozaki, S. (1994) Identification of protein receptor for Clostridium botulinum type $\mathrm{B}$ neurotoxin in rat brain synaptosomes. J. Biol. Chem. 269, 10498-10503.

(30) Nishiki, T. I., Tokuyama, Y., Kamata, Y., Nemoto, Y., Yoshida, A., Sato, K., Sekiguchi, M., Takahashi, M., and Kozaki, S. (1996) The high-affinity binding of Clostridium botulinum type B neurotoxin to synaptotagmin II associated with gangliosides GT1b/GD1a. FEBS Lett. 378, 253-257.

(31) Dong, M., Tepp, W. H., Liu, H., Johnson, E. A., and Chapman, E. R. (2007) Mechanism of botulinum neurotoxin B and G entry into hippocampal neurons. J. Cell Biol. 179, 1511-1522.

(32) Dong, M., Yeh, F., Tepp, W. H., Dean, C., Johnson, E. A., Janz, R., and Chapman, E. R. (2006) SV2 is the protein receptor for botulinum neurotoxin A. Science (Washington, DC, U. S.) 312, 592596.

(33) Mahrhold, S., Rummel, A., Bigalke, H., Davletov, B., and Binz, T. (2006) The synaptic vesicle protein $2 \mathrm{C}$ mediates the uptake of botulinum neurotoxin A into phrenic nerves. FEBS Lett. 580, 20112014.

(34) Dong, M., Liu, H., Tepp, W. H., Johnson, E. A., Janz, R., and Chapman, E. R. (2008) Glycosylated SV2A and SV2B mediate the entry of botulinum neurotoxin E into neurons. Mol. Biol. Cell 19, $5226-5237$.

(35) Fu, Z., Chen, C., Barbieri, J. T., Kim, J. J. P., and Baldwin, M. R. (2009) Glycosylated SV2 and gangliosides as dual receptors for botulinum neurotoxin serotype F. Biochemistry 48, 5631-5641.

(36) Mahrhold, S., Strotmeier, J., Garcia-Rodriguez, C., Lou, J., Marks, J. D., Rummel, A., and Binz, T. (2013) Identification of the SV2 protein receptor-binding site of botulinum neurotoxin type $\mathrm{E}$. Biochem. J. 453, 37-47.

(37) Peng, L., Tepp, W. H., Johnson, E. A., and Dong, M. (2011) Botulinum neurotoxin D uses synaptic vesicle protein SV2 and gangliosides as receptors. PLoS Pathog. 7, No. e1002008.

(38) Rummel, A., Häfner, K., Mahrhold, S., Darashchonak, N., Holt, M., Jahn, R., Beermann, S., Karnath, T., Bigalke, H., and Binz, T. (2009) Botulinum neurotoxins $\mathrm{C}, \mathrm{E}$ and $\mathrm{F}$ bind gangliosides via a conserved binding site prior to stimulation-dependent uptake with botulinum neurotoxin F utilising the three isoforms of SV2 as second receptor. J. Neurochem. 110, 1942-1954.

(39) Brunt, J., Carter, A. T., Stringer, S. C., and Peck, M. W. (2018) Identification of a novel botulinum neurotoxin gene cluster in Enterococcus. FEBS Lett. 592, 310-317.
(40) Strotmeier, J., Willjes, G., Binz, T., and Rummel, A. (2012) Human synaptotagmin-II is not a high affinity receptor for botulinum neurotoxin $B$ and $G$ : Increased therapeutic dosage and immunogenicity. FEBS Lett. 586, 310-313.

(41) Nakamura, K., Kohda, T., Umeda, K., Yamamoto, H., Mukamoto, M., and Kozaki, S. (2010) Characterization of the D/C mosaic neurotoxin produced by Clostridium botulinum associated with bovine botulism in Japan. Vet. Microbiol. 140, 147-154.

(42) Tao, L., Peng, L., Berntsson, R. P. A., Liu, S. M., Park, S., Yu, F., Boone, C., Palan, S., Beard, M., Chabrier, P. E., Stenmark, P., Krupp, J., and Dong, M. (2017) Engineered botulinum neurotoxin B with improved efficacy for targeting human receptors. Nat. Commun. 8, 53. (43) Elliott, M., Favre-Guilmard, C., Liu, S. M., Maignel, J., Masuyer, G., Beard, M., Boone, C., Carré, D., Kalinichev, M., Lezmi, S., Mir, I., Nicoleau, C., Palan, S., Perier, C., Raban, E., Zhang, S., Dong, M., Stenmark, P., and Krupp, J. (2019) Engineered botulinum neurotoxin $B$ with improved binding to human receptors has enhanced efficacy in preclinical models. Sci. Adv. 5, No. eaau7196.

(44) Stenmark, P. P., Dong, M., Dupuy, J. J., Chapman, E. R., and Stevens, R. C. (2010) Crystal structure of the botulinum neurotoxin type $\mathrm{G}$ binding domain: Insight into cell surface binding. J. Mol. Biol. 397, 1287-1297.

(45) Jin, R., Rummel, A., Binz, T., and Brunger, A. T. (2006) Botulinum neurotoxin $\mathrm{B}$ recognizes its protein receptor with high affinity and specificity. Nature 444, 1092-1095.

(46) Findeisen, M., Brand, T., and Berger, S. (2007) A 1H-NMR thermometer suitable for cryoprobes. Magn. Reson. Chem. 45, 175178.

(47) Mayer, M., and Meyer, B. (1999) Characterization of ligand binding by saturation transfer difference NMR spectroscopy. Angew. Chem., Int. Ed. 38, 1784-1788.

(48) Mayer, M., and Meyer, B. (2001) Group Epitope Mapping by Saturation Transfer Difference NMR To Identify Segments of a Ligand in Direct Contact with a Protein Receptor. J. Am. Chem. Soc. $123,6108-6117$.

(49) Keller, R. J. L. CARA: The Computer Aided Resonance Assignment Tutorial. http://cara.nmr-software.org/portal/.

(50) Wishart, D. S., Sykes, D. B., and Richards, F. M. (1992) The Chemical Shift Index: A Fast and Simple Method for the Assignment of Protein Secondary Structure through NMR Spectroscopy. Biochemistry 31, 1647-1651.

(51) Mayo, A., and Kyoko, Y. Empirical analysis of backbone chemical shifts in proteins. http://www.bmrb.wisc.edu/published/ Ikura_cs_study/.

(52) Wishart, D. S., and Nip, A. M. (1998) Protein chemical shift analysis: a practical guide. Biochem. Cell Biol. 76, 153-163.

(53) Willjes, G., Mahrhold, S., Strotmeier, J., Eichner, T., Rummel, A., and Binz, T. (2013) Botulinum neurotoxin G binds synaptotagmin-II in a mode similar to that of serotype B: Tyrosine 1186 and lysine 1191 cause its lower affinity. Biochemistry 52, 3930-3938.

(54) Scholtz, J. M., Qian, H., York, E. J., Stewart, J. M., and Baldwin, R. L. (1991) Parameters of helix-coil transition theory for alaninebased peptides of varying chain lengths in water. Biopolymers 31, $1463-1470$ 